\title{
ARTICULO ORIGINAL-2016: La Valoración de Incapacidades y los Síntomas Físicos Médicamente Inexplicables
}

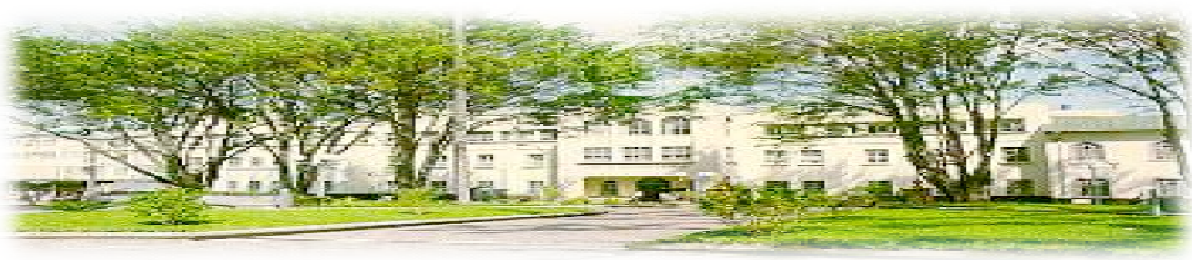

Hospital San Juan de Dios, San José, Costa Rica. Fundado en 1845

\section{ISSN}

2215-2741

Recibido: $12 / 08 / 2015$

Aceptado: 16/11/2015

Grettchen Flores Sandí ${ }^{1}$

\begin{abstract}
${ }^{1}$ Médico Especialista en Medicina Legal. Magister Science en Administración de Centros y Servicios de Salud. Máster en Valoración de Incapacidades y Daño Corporal. Departamento Clínico Medicina Legal, Escuela de Medicina, Universidad de Costa Rica. Correo Electrónico: grettchenflores@medicos.cr
\end{abstract}

\section{RESUMEN}

En la práctica médica diaria, la valoración de capacidad laboral independientemente del campo de actuación del médico evaluador, ya sea por enfermedad o accidente común o laboral, supone un difícil reto que requiere incorporar un análisis de la validez de cada caso dadas las repercusiones personales y sociales. Además los síntomas físicos médicamente inexplicables son frecuentes en las consultas, de alto costo, se asocian a incapacidad laboral, generan frustración al paciente y al médico, pudiendo ser denunciados en relación a contingencias profesionales. Con el objetivo de identificar el procedimiento del médico perito/evaluador para el otorgamiento de incapacidad laboral y parámetros útiles en caso de síntomas físicos médicamente inexplicables, se realizó una revisión descriptiva y se obtuvo que el impacto de estos síntomas en la incapacidad laboral varía de paciente a paciente y que existen pocos estudios en relación con implicaciones laborales. Además el análisis de la validez es una tarea muy compleja que requiere la apreciación de elementos/pruebas de carácter objetivo, que incluyen un adecuado abordaje clínico, comprensión de pruebas diagnósticas complementarias (incluida la valoración psiquiátrica) y de cargas de los puestos de trabajo, contrastada con otras variables de la capacidad funcional para el trabajo como edad, escolaridad, clasificación funcional de acuerdo a baremos, estructura lesionada y pronóstico de la lesión. De ahí que se concluye que a fin de mejorar la validez de los resultados adquieren importancia las habilidades, en lo que respecta a formación médica, conocimientos legales, experiencia, manejo de la información, capacidad de aprendizaje continuo y razonamiento ético. 


\section{PALABRAS CLAVE}

Medicina evaluadora, incapacidad laboral, síntomas físicos médicamente inexplicables, análisis de validez

\section{ABSTRACT}

In daily medical practice, assessment of working capacity regardless of the field of action of the evaluating physician, either by common or occupational disease or accident, is a difficult challenge that requires incorporating an analysis of the validity of each case given the personal and social repercussions. In addition medically unexplained physical symptoms are common in the consultations, high cost, associated with incapability at work, generate frustration to the patient and the doctor, and can be reported in relation to occupational contingencies. In order to identify the procedure of evaluating medicine to grant certificate of incapacity and useful parameters in case of medically unexplained physical symptoms, a descriptive review was conducted and it was found that the impact of these symptoms on incapacity varies from patient to patient and that there are few studies regarding labor implications. Besides the analysis of validity is a very complex task that requires the assessment of matters / objective tests, including an adequate clinical approach, understanding of complementary diagnostic tests (including psychiatric assessment) and job loads, contrasted with other variables of the functional capacity to work as age, education, functional classification according to scales, injured structure and prognosis of the injury. Hence, it is concluded that in order to improve the validity of results become important skills, regarding medical training, legal knowledge, experience, information management, capacity for continuous learning and ethical reasoning.

\section{KEY WORDS}

Evaluating medicine, disability/impairment, medically unexplained physical symptoms, validity analysis

\section{INTRODUCCIÓN}

La práctica diaria de los diversos médicos que intervienen en la valoración de la capacidad laboral está inmersa en continuos cambios de competencias, según el tipo de contingencia que la ocasiona sea por enfermedad o accidente

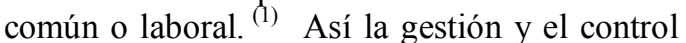
de la incapacidad cuando un trabajador está impedido para realizar su trabajo por padecer una enfermedad o un accidente sea o no laboral, según sea el caso, puede darse por los médicos adscritos a la seguridad social o a las compañías aseguradoras, y en caso de que se interpongan denuncias judiciales termina siendo competencia del médico forense. En cuyo caso una vez establecido el diagnóstico, el parámetro principal es la valoración de la capacidad residual de la persona para reintegrarse al trabajo, establecer las incapacidades temporal y permanente, considerando que desde un punto de vista médico existe flexibilidad dada por la evolución de las lesiones. ${ }^{(2)}$

En este contexto, la valoración del menoscabo constituye un punto importante en el quehacer médico, en lo que respecta al paciente y por la repercusión socioeconómica que conlleva. ${ }^{(3)} \mathrm{El}$ informe médico debe registrar datos legibles, completos, veraces y médicamente correctos, es un acto médico que se ajusta a la buena práctica médica y constituye un documento de calidad que da cuenta de las circunstancias y del estado de salud de una víctima-paciente que necesita junto a la atención clínica una adecuada administración de justicia. ${ }^{(2)}$

Otros conceptos a tomar en cuenta en estas valoraciones son el de Medicina Evaluadora que constituye aquella rama de la medicina que estudia las diferentes enfermedades o trastornos que padece una persona, a efectos de determinar su magnitud y su repercusión sobre diferentes aspectos de la vida de esa persona o de la relación de esta con el entorno social. Y se realiza en distintos ámbitos médicos, no solo el legal. Se encarga de estudiar verificar, cuantificar y describir procesos patológicos, congénitos o adquiridos, tanto físicos como psíquicos, con el fin de determinar su posible repercusión orgánica, funcional, laboral, económica y legal. ${ }^{(3,4)}$ Por su parte la Medicina Evaluadora de Incapacidades Laborales es "aquella rama u orientación de la medicina evaluadora que tiene por objeto apreciar la repercusión del cuadro clínico de un trabajador, a efectos de que se determine la existencia o no de algún tipo y grado de incapacidad laboral", contextualizada como el estado temporal o permanente de una persona que, por accidente o enfermedad, queda mermada en su capacidad laboral, por la imposibilidad de desempeñar las funciones específicas de una actividad ( $u$ ocupación), en consecuencia de alteraciones anatomopsicofisiológicas provocadas por la enfermedad o accidente. Es decir, se considera incapacidad laboral cualquier reducción o falta (resultante de una deficiencia o disfunción: cualquier pérdida o anormalidad de la estructura o función psicológica, fisiológica o anatómica) de la capacidad, para realizar una actividad laboral de una manera que sea considera normal para una persona o esté dentro del espectro considerado normal. ${ }^{(5)}$ Estando incluido 
en el concepto de incapacidad el riesgo de la vida para sí mismo o para terceros, o de agravamiento que la permanencia en la actividad pueda acarrear al trabajador.

Es decir, para poder calificar a un paciente como "incapacitado laboralmente", no basta con que exista una lesión o una alteración de sus estructuras $\mathrm{y} / \mathrm{o}$ funciones corporales (físicas $\mathrm{o}$ mentales), sino que debe darse ineludiblemente la condición de que dicha lesión o alteración impida el desarrollo de su puesto de trabajo, situación que puede ser transitoria (incapacidad temporal) o permanente (incapacidad permanente). ${ }^{(3)}$ La misión de cualquier perito médico evaluador supone entonces un difícil reto en cualquiera de sus campos de actuación y, como lógica consecuencia, en la consulta de valoración de incapacidad debería ser posible incorporar un análisis de la validez de cada caso concreto. ${ }^{(4)}$

Por lo anterior adquiere importancia la valoración de incapacidades laborales en aquellos casos en los cuales no sea posible establecer un diagnóstico preciso y por lo tanto el análisis de la validez no sea un trabajo sencillo.

El objetivo general de la investigación fue identificar el procedimiento del médico evaluador para el otorgamiento de incapacidad laboral y parámetros útiles en caso de síntomas médicamente inexplicables, y los específicos: describir las características asociadas al concepto de síntomas físicos médicamente inexplicables y su repercusión en la medicina evaluadora de incapacidades laborales, identificar parámetros para el análisis de validez en casos de síntomas físicos médicamente inexplicables y plantear habilidades clínicas que el médico evaluador requiera en la búsqueda de validez de los resultados.

\section{METODOLOGÍA}

Se realizó una revisión sobre el tema en la literatura en inglés, español y portugués. Sin embargo, algunos términos fundamentales para este estudio (incluyendo el término MUPS Síntomas físicos médicamente inexplicables por sus siglas en inglés) no están listados como Medical Subject Headings (MeSH) o Descriptores en Ciencias de la Salud (DeCS). Fue necesaria una combinación de estrategias de búsqueda utilizando descriptores/headings establecidos $\mathrm{y}$ otros términos. Se utilizaron los siguientes términos de búsqueda:

- Palabras clave MeSH/DeCS: Incapacidad laboral, peritaje médico, medicina basada en evidencia, medicina evaluadora, valoración daño corporal, análisis de validez. // Incapacidade laborativa, perícia médica, medicina baseada em evidências, mensuração da incapacidade, avaliação do dano corporal, análise de validade. // Disability/Impairment, medical expertise, evidence-based medicine, evaluating medicine, assessment of personal injury damages, validity analysis

- Otros términos importantes: Síntomas físicos médicamente inexplicables, sintomas sin explicación médica. // Sintomas inexplicáveis, sintomas sem explicação médica. // Medically unexplained physical symptoms, medically unexplained symptoms.

Las bases de datos utilizadas fueron Medline, LILACS y SciELO, no se incluyeron tesis o disertaciones. Solamente estudios que estuviesen dentro de un conjunto de requisitos, después de la lectura de los títulos y cuando estuvieran disponibles de los resúmenes: relevancia para los objetivos de la investigación, clasificación, diagnóstico, evolución y/o valoración de síntomas sin explicación médica, capacidad laboral y procedimiento de valoración en medicina evaluadora, disponibles a textos completos y publicados entre 2010 y 2015. Después de la recuperación de los estudios utilizando estos criterios se excluyeron: estudios de revisión general informativa sobre el tema (se mantuvieron las revisiones sistemáticas y las revisiones conceptuales críticas), relato de casos sin discusión crítica, estudio en niños y adolescentes, estudio de instrumentos e intervenciones de tratamiento, excepto cuando incluyeran información conceptual, estudios que versaran exclusivamente sobre síntomas psiquiátricos médicamente inexplicables, estudios de costos de las intervenciones de salud y estudios disponibles en otros idiomas.

\section{LOS SÍNTOMAS FÍSICOS MÉDICAMEN- TE INEXPLICABLES}

Inicialmente se debe recordar que un síntoma es en medicina la manifestación subjetiva de una enfermedad, siendo el signo la manifestación objetiva. Los síntomas son necesarios para el quehacer médico, la información que dan provienen en realidad de dos sujetos: de aquél que informa (paciente) y de aquél que interpreta la información presuponiendo la honestidad del informante (médico). En el más simple de los casos, la información que da un signo procede de un solo sujeto: el médico. ${ }^{(6)}$ Tradicionalmente, la formación médica se ha centrado en la comprensión y el tratamiento de los síntomas individuales con sus correspondientes hallazgos que conducen a un diagnóstico específico. Sin 
embargo, existen casos en que los pacientes presentan varias quejas no específicas sin los correspondientes signos clínicos o hallazgos de práctica general. $^{(7)}$

En este sentido, los síntomas físicos médicamente inexplicables (MUPS) se definen como síntomas físicos que conducen al paciente a buscar atención de salud, y después de la evaluación clínica, exámenes apropiados, diagnóstico diferencial e interconsultas, no parecen explicarse por una causa claramente definida o el diagnóstico de una enfermedad médica definida (no se establece ninguna base física para los síntomas, no hay evidencia de alteración de la estructura o función del sistema físico ni órgano de que se trate). ${ }^{(4,8-10)}$

En la práctica clínica, de hecho muchas quejas físicas permanecen sin explicación médica, debido a la falta de una causa obvia o cambios patológicos en el examen físico y pruebas de diagnóstico. (11) Ya en el 2002 Escobar JI, Hoyos-Nervi C y Gara M plantearon las siglas MUPS para denominar los síntomas físicos médicamente inexplicables. ${ }^{(4)}$

Un punto a considerar es que además de ser comunes, estos síntomas se han asociado con trastornos mentales en diversos contextos médicos, especialmente en el primer nivel de atención médica y en la población general, pueden presentarse de forma muy variada: desde conjuntos de pocos síntomas autolimitados a patrones de presentación crónicos. ${ }^{(11-13)}$ Los pacientes suelen presentar síntomas complejos como problemas digestivos, dolores musculares, o astenia generalizada, a menudo en combinación; muchos pacientes con incapacidad por razones músculo-esqueléticas o mentales sufren de condiciones que se pueden clasificar como MUPS. (7) $\mathrm{Y}$ aunque los estudios sistemáticos sugieren que estos pacientes tienen una alta comorbilidad psiquiátrica, deterioro funcional grave y una pobre calidad de vida, rara vez son valorados en el segundo nivel de atención por especialistas en psiquiatría; además de que normalmente los pacientes con MUPS se ven a sí mismos como físicamente enfermos por lo que se resisten a la derivación psicológica/psiquiátrica y presentan altas tasas de desempleo e incapacidad laboral. $(10,14)$

Los términos psicógeno, funcional y psicosomático, se utilizan en presencia de una manifestación patológica cuyo origen no se encuentra en una lesión o alteración orgánica, sino en un trastorno psíquico, o cuya aparición y desarrollo se ven influenciados de manera importante por variables psicológicas. ${ }^{(14)} \mathrm{Al}$ respecto hay que tener presente que la somatización como tal no es un diagnóstico psiquiátrico, sino un denominador común de una serie de procesos patológicos agrupados según el DSM-IV (manual diagnóstico y estadístico de los trastornos mentales) como trastornos somatomorfos, que son un conjunto de patologías cuyo diagnóstico se realiza por la presencia de síntomas corporales que sugieren un trastorno físico sin causa orgánica demostrable o mecanismo fisiológico conocido que los explique completamente y por la presunción razonable de que dichos síntomas están asociados a factores psicológicos o estrés. Los pacientes con trastornos somatomorfos crónicos y graves (sobre todo el trastorno por somatización, la hipocondría, la dismorfofobia y el dolor psicógeno) suelen presentarse también con trastornos de la personalidad que determinan la evolución o, incluso, son el diagnóstico principal del caso. ${ }^{(12)}$

Una aclaración al respecto como parte del diagnóstico diferencial, es que los síntomas físicos son reconocidos como parte de síndromes psiquiátricos. Por ejemplo, ellos comúnmente coexisten con signos y síntomas psicológicos en trastorno depresivo mayor; forman parte de los criterios diagnósticos para la ansiedad generalizada, son a menudo vinculados con la psicosis y así sucesivamente. La psiquiatría es una forma legítima de la medicina que tiene un marco de diagnóstico para la enfermedad, por lo tanto un síntoma psiquiátrico explicado, no es médicamente inexplicable. Sin embargo, el diagnóstico psiquiátrico no debe ser por exclusión; sino que debe basarse en los resultados positivos en el contexto de una evaluación integral. ${ }^{(15)}$

Además aproximadamente el $60 \%$ de los pacientes con MUPS tiene un diagnóstico de comorbilidad no somatomorfo. ${ }^{(9)}$ En el caso de las comorbilidades, los síntomas inexplicables compensan la pérdida de función causada por síntomas explicados médicamente, que a su vez se han asociado con altos costos, tanto directos (por su atención), como indirectos (pérdida de productividad debido a ausencia por enfermedad). ${ }^{(7,16)}$ Estos pacientes tienden a tener alta utilización de los servicios y de las pruebas de laboratorio potencialmente innecesarias y consultas, con el aumento de los costos y las altas tasas de complicaciones iatrogénicas. ${ }^{(9)}$

Sin embargo, a pesar de tener altos niveles de angustia psicosocial, la utilización de servicios de salud e incapacidad médica, los pacientes con MUPS a menudo son tratados subóptimamente. Los factores que podrían contribuir a esto incluyen: la identificación inadecuada, el sesgo en el diagnóstico y tratamiento, el defi- 
ciente seguimiento de las referencias y la ausencia de pautas de tratamiento. ${ }^{9}$ Pues existen limitaciones de 'diagnóstico' de estos síntomas como una entidad, más bien es un diagnóstico de exclusión ${ }^{(15)}$

El diagnóstico de los MUPS se realiza mediante distintos nombres - la mayoría inespecíficos- en función de la especialidad médica en que se hace la consulta, aunque el solapamiento clínico es lo habitual ${ }^{4}$ Debido a la falta de consenso en cuanto a la definición, la investigación sobre los aspectos relacionados con el MUPS constituye un reto. Los términos: síntomas físicos médicamente inexplicables, síntomas sin explicación médica, el trastorno somatomorfo, somatización, y los síndromes somáticos (por ejemplo, el síndrome de intestino irritable, la fibromialgia, cistitis intersticial, fatiga crónica, síndrome de agotamiento profesional, etc.) a menudo se utilizan indistintamente. ${ }^{(8-10,16,17)}$ Otros términos relacionados son Trastorno de distress corporal, Síndrome funcional somático y Quejas subjetivas de salud. (7) Esta inconsistente nomenclatura crea dificultades de clasificación porque varios de estos términos asumen una etiología diferente para los síntomas físicos del paciente (es decir, médicos vs psiquiátricos). ${ }^{(9)}$

Una forma de categorizarlos es por síndromes somáticos, ${ }^{(4,12)}$ tal como muestra la tabla I.

Tabla I.

Síndromes somáticos de acuerdo a especialidad médica

\begin{tabular}{|l|l|}
\hline Especialidad Médica & Síndrome Somático \\
\hline Cardiología & Dolor precordial atípico \\
\hline Gastroenterología & Colon irritable, dispepsia no ulcerosa \\
\hline Medicina general & Síndrome de fatiga crónica \\
\hline Neumología & Síndrome de hiperventilación \\
\hline Neurología & Cefalea tensional, acúfenos \\
\hline Ortopedia & Dolor lumbar crónico \\
Otorrinolaringología & Globus faríngeo, tinnitus \\
\hline Reumatología & Fibromialgia \\
\hline Urología/Ginecología & Dolor pelviano crónico \\
\hline
\end{tabular}

Por su parte Huang y McCarron (9) intentan categorizar pacientes con MUPS con base en la severidad y duración de los síntomas, así como las comorbilidades médicas y/o psiquiátricas, ya que explican que el examen médico puede ser limitado o extenso, puede evolucionar con el tiempo (por ejemplo, el diagnóstico se puede hacer dos años después de la aparición de los síntomas primarios), y, a menudo implica la colaboración entre varios especialistas.

Por otro lado, las condiciones que se clasifican como "inexplicables"' contribuyen significativamente a la ausencia laboral por enfermedad, y por lo tanto podrían ser percibidas como un asunto de medicina ocupacional y ambiental. ${ }^{(8)}$

En cuanto a la etiología, hay que tener presente que si bien los síntomas físicos típicamente se explican por: fisiopatología médica, psicopatología, o etiología desconocida. ${ }^{(9)}$ Las causas de MUPS son aún poco conocidas, pero es probable que su etiología sea multifactorial. (11) $\mathrm{Di}$ versos autores citan entre los factores asociados a los MUPS los factores genéticos, biológicos y biopsicosociales. ${ }^{(6,9,12,17,18)}$ También en la historia natural de los MUPS se han descrito factores predisponentes, precipitantes y de perpetuación. (4) Se ha sugerido que los MUPS pueden ser causados por un mecanismo común con diferentes expresiones, explicado por el modelo biopsicosocial o la Teoría de la Activación Cognitiva del Estrés, ${ }^{(7)}$ que se ha estudiado como paradigma en medios laborales, por la respuesta de estrés mediante registros psicofisiológicos, neuroendocrinos, psicoinmunológicos, comportamentales y de bioquímica cerebral. ${ }^{(19)}$

De acuerdo con McGorm y otros, ${ }^{(20)}$ este fenómeno es digno de mayor atención debido a que estos pacientes es poco probable que se beneficien con referencias repetidas a servicios médicos especializados diseñados para encontrar o descartar la enfermedad en lugar de manejar los síntomas; las referencias repetidas consumen recursos diagnósticos limitados a poco beneficio y estos pacientes son propensos a tener problemas tratables como la ansiedad y la depresión que sería susceptible de enfoques alternativos para su atención. Cuando sea posible, puede ser 
útil aclarar la sintomatología como: principalmente somática (expresión de enfermedad psicológica a través de medios físicos), principalmente psiquiátrica (enfermedad psiquiátrica que presentan síntomas físicos) o limítrofe entre somática y psiquiátrica. ${ }^{(9)}$ Otro de los aspectos clave es la frecuente negación o minimización de los elementos psicoemocionales del caso por parte del paciente, que considera que suponen una "deslegitimación" de sus síntomas con respecto a la enfermedad orgánica, ${ }^{(4,14)}$ que puede llevar a un comportamiento defensivo en consultas médicas, desencadenar sentimientos de ansiedad o incluso hostiles en sus médicos. Los médicos también pueden sentir una sensación de desesperanza y frustración. ${ }^{(10,18)}$

Con respecto a la incapacidad laboral el impacto de los síntomas físicos sin explicación médica varía de paciente a paciente. Algunos continúan trabajando como de costumbre, mientras que otros se enferman por síntomas aparentemente comparables. La transición de las quejas a síntomas incapacitantes no es bien conocida. A pesar de esto sólo un pequeño número de estudios sobre los fenómenos asociados a la incapacidad laboral en MUPS ha sido realizado. ${ }^{(7)}$

La capacidad funcional tiene por sí misma una gran importancia ya que constituye, la principal preocupación de los pacientes que se hallan limitados en la realización de aquellas actividades de la vida diaria que consideran fundamentales. La frustración que ello provoca se ve agravada por la incomprensión del entorno motivada por la dificultad de objetivar ese deterioro funcional, a diferencia de otras enfermedades como la artritis reumatoide o la artrosis en las cuales, el deterioro funcional, parece más justificado por la existencia de alteraciones orgánicas demostrables. Otra de las consecuencias del deterioro funcional es la pérdida de jornadas laborales con sus repercusiones económicas asociadas, por ejemplo los pacientes con fibromialgia tienen una tasa de incapacidad laboral cuatro veces mayor que la del resto de los trabajadores. ${ }^{(21)}$ De hecho según la Organización Mundial de la Salud el dolor crónico afecta a un número importante de personas, y es una causa importante de incapacidad física para trabajar, los estudios muestran que el $10 \%$ de la población sufre de dolor difuso crónico, de los cuales $5.03 \%$ son casos de fibromialgia, descrito como la enfermedad crónica más común de dolor actualmente. ${ }^{(22)}$

En el análisis de estos casos, el médico evaluador de incapacidades debe tener presente que con frecuencia, además, estos pacientes presentan un estrés psicosocial importante cuando acuden al médico y problemas de ajuste social que muchas veces tienen un refuerzo ambiental de los síntomas. Las ganancias derivadas de la enfermedad y de la asunción del papel de enfermo, los mecanismos cognitivo-perceptivos anómalos y los trastornos de la relación médicopaciente, son factores imprescindibles a considerar para entender la queja del paciente. ${ }^{(12)}$ Esto se explica ya que la enfermedad y la discapacidad son conductas sujetas al aprendizaje y la influencia de los refuerzos positivos y negativos. Así, los principios del condicionamiento operante contribuyen a explicar por qué los pacientes que reciben prestaciones económicas, u otras ventajas, tienden más a solicitar incapacidad permanente o los lesionados de accidentes de tránsito en países que no incluyen una indemnización vinculada a las secuelas, presentan una menor proporción de determinados cuadros dolorosos crónicos como secuela. En este sentido, se usa el término "sistemas de mantenimiento del estado de enfermedad" para hacer referencia a los factores biopsicosociales que favorecen que una enfermedad o limitación se perpetúe. ${ }^{(4)}$

La existencia de un contexto médico-legal como el descrito, que proporciona acceso a prestaciones económicas, implica considerar la validez en su sentido de firmeza, consistencia y valor legal- de las consultas de valoración de daño corporal, analizando la posible distorsión orientada a obtener o prolongar prestaciones, sea de manera fraudulenta o no. Estas situaciones, además de graves implicaciones en el ámbito económico que lesionan el principio deontológico de Justicia, suponen un elevado riesgo de generar daño al paciente, reforzando unos roles de enfermedad e invalidez que no se justifican por la patología que presentan, lo que iría en contra de los principios de Beneficencia y No maleficencia, pudiendo llevar a situaciones de dependencia injustificada, lesionando incluso el principio de autonomía.

\section{LA PERICIA MÉDICA EVALUADORA DE INCAPACIDADES LABORALES Y VALI- DEZ DE CONSTRUCTO}

La expedición de la incapacidad es, en sí misma, un acto pericial del médico, y como tal conlleva una responsabilidad ética y legal inherente al ejercicio de la profesión. ${ }^{(23)}$ Para estar ante una situación de incapacidad laboral deben de darse lesiones de tal entidad que causen limitación y restricciones funcionales intensas y limitaciones que puestas en relación con trabajo causan impedimento para el trabajo o imposibilidad laboral. ${ }^{(24)}$ Es decir, valorar una situación de Inca- 
pacidad Laboral es poner en relación las capacidades perdidas o las que mantiene el trabajador con las capacidades requeridas por el trabajo, las capacidades que precisa el trabajo.

En relación con la incapacidad laboral asociada a contingencias profesionales, es importante conocer el significado de los términos utilizados, en Costa Rica de acuerdo con Ley No. 6727 de 24 de marzo de 1982- Publicada en La Gaceta No. 57 de 24 de marzo de 1982, en su artículo 223, que establece que los riesgos del trabajo pueden producir al trabajador incapacidad temporal y permanente, definidas como se muestra en la Tabla II.

Tabla II

Criterios de Incapacidad de acuerdo con el artículo 223 de la Ley No. 6727 de 24 de marzo de 1982

Incapacidad temporal: constituida por la pérdida de facultades o aptitudes que imposibilita al
trabajador para desempeñar el trabajo por algún tiempo. Esta incapacidad finaliza por:
- $\quad$ Declaratoria de alta, al concluir el tratamiento.
- Haber transcurrido un plazo de dos años a partir de la ocurrencia del riesgo, sin haber
cesado la incapacidad temporal del trabajador, se procederá a establecer el porcentaje de
incapacidad permanente, y se suspenderá el pago del subsidio, sin perjuicio de que se
puedan continuar suministrando las prestaciones médico-sanitarias y de rehabilitación al
trabajador.
- Abandono injustificado de las prestaciones médico-sanitarias que se le suministran.
- Muerte del trabajador.
Incapacidad permanente: se establecen grados en función de la repercusión en la capacidad labo-
ral: $\quad$ Incapacidad menor permanente: es la que causa una disminución de facultades o aptitudes
para el trabajo, consistente en una pérdida de capacidad general, orgánica o funcional, que
va del $0,5 \%$ al $50 \%$ inclusive.
Incapacidad parcial permanente: es la que causa una disminución de facultades o aptitudes
para el trabajo consistentes en una pérdida de capacidad general, orgánica o funcional
igual o mayor al $50 \%$ pero inferior al $67 \%$.
Incapacidad total permanente: es la que causa una disminución de facultades o aptitudes
para el trabajo, consistente en una pérdida de capacidad general, orgánica o funcional,
igual o superior al $67 \%$.
Gran invalidez: ocurre cuando el trabajador ha quedado con incapacidad total permanente
y además requiere de la asistencia de otra persona, para realizar los actos esenciales de la
vida: Caminar, vestirse y comer.

Al respecto la imposibilidad para asistir a trabajar por causa de un problema de salud que requiere reposo como parte de la terapéutica, debe ser evaluada desde el punto de vista temporal y se pondera acorde a los tiempos de sanación conocidos por cada médico. (2) Sin embargo, debe tenerse en cuenta que su determinación solo con esta consideración es difícil, pues la incapacidad temporal es el resultado de parámetros médicos (lesiones y sus repercuciones funcionales, evolución médica, características del trabajo) y no médicos (laborales y administrativos, personales, familiares), estos últimos (no médicos) pueden modificar e incrementar los días totales de la incapacidad laboral temporal, alterando la situación real respecto a este daño. Al respecto Navarro, ${ }^{(1)}$ cita como parámetros:

- Personales y Socio-Familiares: la personalidad y características del trabajador hacen que la misma dolencia impida o no realizar un mismo trabajo.
A veces la necesidad del cuidado de hijos o de familiares dependientes pueden alargar los procesos.

- Sanitarios: las lista de espera de los servicios, la sobrecarga de los médicos del primer nivel de atención, la ausencia de comunicación entre el primer nivel y atención especializada, la insuficiente cumplimentación de las boletas de incapacidad, etc., lo que hace que a veces se alargue el proceso $\mathrm{y}$ no se disponga de toda la información médica necesaria para valorar bien la situación.

- Otros:

a. Dificultad para compartir la información de los procesos. La falta de conexión informática que proporcione información útil, hace que no se compartan datos con el resto de los agentes implicados, lo que impide desarrollar 
eficazmente las funciones encomendadas. No hay información suficiente sobre procesos o decisiones anteriores del resto de las instituciones de salud. Se adelantan pruebas o tratamientos que se repiten duplicando el gasto de forma innecesaria y sobrecargando las listas de espera.

b. Falta de eficacia de las herramientas de control: Existe un porcentaje excesivo de propuestas de alta que no se contestan y a veces las contestaciones no se hallan suficientemente motivadas desde el punto de vista médico.

c. La dispersión normativa: $\mathrm{Da}$ lugar a problemas interpretativos y lagunas de la legislación.

Por su parte la valoración de la incapacidad permanente, consiste en el análisis descriptivo del daño secuelar, las exigencias psicofísicas del puesto de trabajo y la aptitud laboral residual respecto a su trabajo y otros trabajos. Este concepto contempla que un mismo grado de afectación funcional pueda tener consecuencias diferentes entre los individuos, dependiendo de otras variables como el sexo, la edad o el tipo de trabajo. Por ejemplo, un paciente con una EPOC moderada puede ser capaz de realizar un trabajo de oficina pero ser subsidiario de una incapacidad si trabaja como bombero. Para establecer este concepto se necesita conocer la enfermedad y el análisis del puesto de trabajo. Contempla también un aspecto legal o administrativo y se acompaña de una prestación económica. ${ }^{(25)}$

Ante las repercusiones médicas, legales, sociales y económicas de la incapacidad laboral, adquiere relevancia la práctica de la medicina basada en la evidencia con la integración de la mejor evidencia científica, la pericia clínica individual y los valores de los pacientes. El poder deductivo asociado a la pericia clínica no se fundamenta solo en los años de experiencia, sino también en la observación y el estudio continuados, el rigor en la obtención de datos y el sentido común. En caso de síntomas sin explicación médica, el abordaje de la valoración del dolor como incapacitante laboral es extremadamente difícil por la necesidad de objetivar y dimensionar las limitaciones derivadas del dolor y por la necesidad de ponerlas en relación con las capacidades requeridas por el trabajo. Así mismo valorar la servidumbre terapéutica o el impacto que pueda ocasionar la analgesia en el desarrollo de su trabajo. Cuestión más complicada es diferenciar la exageración o el sobredimensionamiento clínico del paciente, y más aún el componente simulador. No se puede hablar de dolor en singular, pues existen múltiples tipos de dolores y además cada uno de ellos se manifiesta de forma diferente, dependiendo de las características y condiciones del proceso causal y del propio sujeto. (24)

Aunque la validez de criterio es una propiedad psicométrica fundamental de cualquier instrumento de medida que disponga de un patrón oro (gold standard) con el que compararlo, en el caso de la capacidad funcional, lo mismo que en el caso del dolor o la calidad de vida, no se dispone de ninguna prueba que pueda considerarse de esta manera. En estos casos cobra especial relevancia la "validez de constructo", cuyo estudio implica definir a priori el constructo a evaluar, en este caso capacidad funcional, en función de su relación con otras variables. ${ }^{(21)}$

En este sentido se deben evaluar de forma conjunta las alteraciones anatómicas y la repercusión en la capacidad funcional, siempre mediante la utilización de métodos objetivos y con resultados reproducibles, adoptando criterios de flexibilidad para casos específicos. No obstante, de acuerdo con Domínguez y otros, ${ }^{(4)}$ el análisis de la validez en el ámbito de la medicina evaluadora es una tarea muy compleja que actualmente no cuenta con un método estructurado, quedando a criterio de cada perito médico la atención que presta a éste asunto y las conclusiones que obtiene al respecto.

La validez en medicina evaluadora vendría dada por la estabilidad, seguridad y valor legal de las pruebas disponibles en la valoración médica.

\section{LA METODOLOGÍA DE LA MEDICINA EVALUADORA DE INCAPACIDADES}

En la actualidad se utilizan métodos convencionales, basados en sus experiencias profesionales y conocimientos sobre las enfermedades, de manera observacional para cuantificar la capacidad funcional de un paciente. En el campo forense debido a la gran responsabilidad para la decisión judicial, se convierte en esencial tener un instrumento científicamente probado para dar seguridad y eficiencia en los informes médicos. ${ }^{(26)}$

En este sentido, la valoración de la capacidad laboral comprende en primer lugar la evaluación de la deficiencia que debe realizar el médico evaluador y es el principal objeto de esta normativa. Incluye el diagnóstico de enfermedad, la valoración de la gravedad y de su impacto en la capacidad laboral. Es importante considerar el efecto del puesto de trabajo en la evolución de 
la enfermedad, tanto como factor causal o como factor de riesgo de mala evolución o agravamiento. Otro factor a incluir en esta evaluación es el pronóstico y la dificultad que añade mantener determinados tratamientos. ${ }^{(25)}$

De acuerdo con Vicente, ${ }^{(24)}$ la valoración de la capacidad laboral supone determinar la existencia de una lesión o proceso patológico (determinar las deficiencias y objetivar sus consecuencias que son las limitaciones orgánicas y/o funcionales que origina en el trabajador), conocer las tareas realizadas por el trabajador (conocer los requerimientos profesionales y circunstancias específicas del ambiente laboral), establecer la relación entre las limitaciones del trabajador y los requerimientos del trabajo y concluir si está o no incapacitado.

Se debe acudir a la apreciación de elementos de carácter objetivo y de una forma didáctica, se pueden clasificar los principales métodos de actuación en medicina evaluadora en métodos negativos y positivos, ${ }^{(3,24)}$ tal y como se muestra en la Tabla III

Tabla III

Elementos de análisis en medicina evaluadora

\begin{tabular}{|l|l|}
\hline \multicolumn{1}{|c|}{ Elementos Objetivos } & \multicolumn{1}{c|}{ Métodos de actuación } \\
\hline Historia Clínica: Anamnesis correcta. \\
Exploración Física: Exploración Funcional \\
Específica. \\
$\begin{array}{l}\text { Pruebas: Exploratorias Complementarias. } \\
\text { Tratamiento: Valoración Terapéutica, a } \\
\text { través de documentos médicos de la } \\
\text { asistencia del paciente, para conocer } \\
\text { antecedentes, evolución, hallazgos, estado } \\
\text { actual. }\end{array}$ & $\begin{array}{l}\text { Métodos negativos: La valoración se basa en } \\
\text { una serie de listados que indican enfermedades } \\
\text { y características de las mismas que impiden la } \\
\text { realización de alguna profesión u oficio (reque- } \\
\text { rimiento de permisos o licencias). } \\
\text { Métodos positivos: La valoración consiste en } \\
\text { medir la capacidad funcional de la persona. } \\
\text { Básicamente se pueden resumir según tres } \\
\text { metodologías de trabajo en: } \\
\text { Descriptivos: se basan en la descrip- } \\
\text { ción y valoración subjetiva del médi- } \\
\text { co evaluador, sobre las lesiones pre- } \\
\text { sentes. } \\
\text { Baremos: identifican determinadas } \\
\text { lesiones o enfermedades con una can- } \\
\text { tidad indemnizatoria o con puntua- } \\
\text { ción (puntos o porcentaje). } \\
\text { Métodos: aplican una determinada } \\
\text { sistemática de exploración y valora- } \\
\text { ción de cada caso. }\end{array}$ \\
\hline
\end{tabular}

Son susceptibles de causar una incapacidad permanente aquellas condiciones crónicas que a pesar de haber recibido un tratamiento adecuado condicionen una pérdida mantenida de función. Esta condición debe estar acreditada mediante pruebas objetivas y al menos debe comprender precisamente la realización de historia clínica, historia laboral, exploración física y pruebas complementarias que pueden ayudar a la evaluación y el diagnóstico en el momento de la valoración. Por ejemplo, la termografía infrarroja ha traído más objetividad para el diagnóstico de fibromialgia $\mathrm{y}$, además proporciona más información para el médico, de manera que puede correlacionar los hallazgos clínicos con la capacidad funcional y evaluar la presencia de la conexión causal con el trabajo. Además, otros síntomas de las mismas condiciones patológicas pueden ser excluidos por estas pruebas, proporcionando una mayor precisión en el diagnóstico.
$(22,25)$ En el caso de los síntomas sin explicación médica, para poder establecer el diagnóstico diferencial con otras patologías adquiere relevancia la interconsulta a psiquiatría como prueba complementaria.

Por otro lado, la historia laboral debe ser exhaustiva, reseñando las características del puesto de trabajo, la demanda energética que supone, las condiciones climáticas en que se realiza, la exposición a agentes irritantes y los horarios con posible turnicidad. ${ }^{(25)}$

Veronesi, ${ }^{(26)}$ propone considerar como variables para evaluar la capacidad funcional para el trabajo: edad, escolaridad, clasificación funcional de acuerdo a baremos, estructura lesionada y pronóstico de la lesión, según se muestra en la Tabla IV; y concluye que la formación específica del médico evaluador es fundamental para 


\section{Variables para evaluar la capacidad para el trabajo}

\begin{tabular}{|c|c|}
\hline Variable & Concepto \\
\hline Edad: & $\begin{array}{l}\text { se estableció para esta variable como parámetro, la edad inicial permitida para } \\
\text { trabajar y la edad de retirada definitiva por la Seguridad Social, donde cuanto } \\
\text { más viejo mayor la incapacidad, una condición científicamente probada y de } \\
\text { conocimiento notorio. }\end{array}$ \\
\hline Escolaridad: & $\begin{array}{l}\text { hoy en día la educación es un factor clave para las oportunidades de trabajo, si } \\
\text { se asocia con el entrenamiento funcional específico, el campo de trabajo } \\
\text { aumenta más. Para la teoría del capital humano, cuanto mayor es el nivel } \\
\text { educativo, mayor es la oportunidad en el mercado laboral, esto dentro de un } \\
\text { entorno económico equilibrado, dándose una relación causal (educación } \\
\text { explicando las oportunidades). }\end{array}$ \\
\hline $\begin{array}{l}\text { Baremo de clasifica- } \\
\text { ción de la funciona- } \\
\text { lidad: }\end{array}$ & $\begin{array}{l}\text { es un instrumento utilizado para clasificar la capacidad funcional, que se divide } \\
\text { en cinco categorías en función de su incapacidad. }\end{array}$ \\
\hline $\begin{array}{l}\text { Estructura lesiona- } \\
\text { da: }\end{array}$ & cuanto mayor es el número de estructuras afectadas mayor será la discapacidad \\
\hline $\begin{array}{l}\text { Pronóstico de la } \\
\text { lesión: }\end{array}$ & $\begin{array}{l}\text { de acuerdo a los parámetros de daño corporal ante las valoraciones de } \\
\text { indemnización, puede tener la condición de Incapacidad Temporal o } \\
\text { Permanente }\end{array}$ \\
\hline
\end{tabular}

En la valoración además debe considerarse la probabilidad que se presenten pacientes con síntomas de etiología orgánica poco clara o psicosomática. En este sentido, la evaluación debe considerar la limitación del paradigma del modelo biomédico que considera que cualquier enfermedad se reduce a una causa (agentes patógenos externos: virus, infecciones, etc. o agentes patógenos internos: desequilibrio bioquímico) que se busca identificar y modificar; se debe considerar al paciente en sus dimensiones psicológicas y somáticas. Además, estos síntomas inexplicables desde un punto de vista médico, son muchas veces más crónicos y más refractarios que muchos de los síntomas con base orgánica. (14) En la evaluación de un paciente con síntomas físicos médicamente inexplicables, se debe descartar la presencia de un trastorno orgánico, evaluar la existencia de trastornos psiquiátricos y buscar un diagnóstico positivo de somatización.. ${ }^{(12)}$

El examen físico es importante para descartar causas orgánicas y con el objetivo de que el paciente se sienta "revisado". Cuando se evalúa a pacientes con probables somatizaciones, el médico debe considerar patologías cuya aparición clínica es vaga y multisistémica. Al considerar síntomas neurológicos como parestesias o debilidad, y dependiendo del contexto, es mucho más probable una somatización que formas raras de esclerosis múltiple, síndromes miasténicos o síndromes paraneoplásicos. También es recomendable ser conservador con la solicitud de exámenes complementarios, que, si bien pueden servir como reaseguro, conducen al aumento del riesgo de que se obtengan resultados falsamente positivos, probabilidad que aumenta en función del número de pruebas solicitadas. Hay que recordar que el valor predictivo positivo (VPP) de una prueba depende de la prevalencia de la condición clínica que se está pesquisando, por lo tanto el VPP tenderá a ser bajo cuando se están solicitando pruebas diagnósticas para "descartar" diagnósticos poco probables. ${ }^{(12)}$

Ante estos casos hay que tener en cuenta que al solicitar una prueba invasiva a un insistente paciente con MUPS que no presenta criterios para ello, se favorece que interprete que su cuadro es grave y requiere estudios porque se desconoce su causa; mantener una incapacidad laboral sin un motivo que lo justifique supone reforzar su conducta y confirmar una discapacidad significativa. Enviar al paciente esos mensajes convierte al médico evaluador en parte del problema. $^{(4)}$

Otro punto importante en la valoración de estos pacientes es que muchas enfermedades comparten signos y síntomas; a su vez, muchas manifestaciones clínicas no necesariamente representan enfermedad. La entrevista, el examen físico y los exámenes complementarios reducen el enorme conjunto de posibilidades a un pequeño número de diagnósticos diferenciales, de acuerdo con Guzmán, ${ }^{(12)}$ las enfermedades con las 
que se hace diagnóstico diferencial se citan en

la Tabla V

Tabla V

Diagnósticos diferenciales de Síntomas Físicos Médicamente Inexplicables

\begin{tabular}{|c|}
\hline Diagnósticos Diferenciales \\
\hline - Malos tratos domésticos y abuso sexual. \\
\hline - Trastornos adaptativos. \\
\hline - Personalidad esquizoide. \\
\hline - Personalidad histriónica. \\
\hline $\begin{array}{l}\text { - Simulación: el síntoma suele tener un tinte burdo o falso, el beneficio es groseramente visible y el } \\
\text { paciente tiene antecedentes de simulación. }\end{array}$ \\
\hline - Alcoholismo y otras dependencias. \\
\hline $\begin{array}{l}\text { - Trastornos ansiosos y depresivos que pueden presentarse con síntomas funcionales somáticos } \\
\text { secundarios. Especialmente en presuntas somatizaciones que se manifiestan después de los } 40 \text { años. }\end{array}$ \\
\hline $\begin{array}{l}\text { - Esquizofrenia o en depresiones psicóticas que pueden presentar síntomas somáticos funcionales } \\
\text { que suelen presentar características bizarras o extravagantes, ser menos numerosos y más persisten- } \\
\text { tes y estables. }\end{array}$ \\
\hline $\begin{array}{l}\text {-Otros trastornos somatomorfos: En la hipocondría el síntoma dominante es la preocupación o la } \\
\text { convicción de padecer una enfermedad grave (más que los propios síntomas), se demandan al médi- } \\
\text { co más exploraciones diagnósticas que tratamiento y la personalidad del paciente suele ser menos } \\
\text { disemocional y más obsesiva o controlada que en los trastornos por somatización. Los síntomas } \\
\text { conversivos y los dolores somatomorfos pueden formar parte del trastorno por somatización pero, a } \\
\text { diferencia del trastorno conversivo y del trastorno por dolor somatomorfo, están acompañados por } \\
\text { otros síntomas funcionales. }\end{array}$ \\
\hline $\begin{array}{l}\text { - Trastornos facticios: Los síntomas los producen voluntariamente los pacientes, sin otro beneficio } \\
\text { aparente que el de ser considerados y tratados como enfermos o ingresar en hospitales. Los afectados } \\
\text { suelen presentar trastornos por personalidades disemocionales (grupo B del DSM-IV). Se presenta } \\
\text { más en personas que sufrieron abusos o deprivaciones importantes en la infancia y en el personal } \\
\text { sanitario. }\end{array}$ \\
\hline $\begin{array}{l}\text { - Enfermedades orgánicas sistémicas que cursan con una afectación general importante, como el } \\
\text { hipertiroidismo, el hiperparatiroidismo, el lupus eritematoso sistémico, la miastenia gravis, la porfi- } \\
\text { ria aguda intermitente, la esclerosis múltiple y algunos procesos infecciosos o expansivos del siste- } \\
\text { ma nervioso central. }\end{array}$ \\
\hline $\begin{array}{l}\text { - Otras enfermedades psiquiátricas, como depresión, ataques de pánico o trastorno delirante, pueden } \\
\text { simular en una fase inicial un trastorno somatoforme. }\end{array}$ \\
\hline
\end{tabular}


Para Jutel, ${ }^{(15)}$ la interpretación clínica en lugar de diagnóstico clínico es una manera de reconciliar la medicina y el individuo, el síntoma inexplicable y la angustia del paciente. La medicina debe orientarse a la claridad y la objetividad, debe permitir que el paciente cuente su padecimiento aun en ausencia de patología orgánica y permitir un marco alternativo para explicar su queja. Utilizando el diagnóstico como uno de muchos posibles resultados en medicina.

Considerando la posibilidad de comorbilidad psiquiátrica y los posibles diagnósticos diferenciales en la esfera mental, adquiere importancia en estos casos la valoración psiquiátrica de previo a ser valorados por el médico evaluador de incapacidades o en caso contrario referir al paciente a valoración especializada cuando el médico lo considere como prueba complementaria.

\section{CONCLUSIONES}

Con base en lo anterior, si bien en la práctica de la medicina evaluadora de incapacidades como en la del resto de especialidades involucradas en la valoración de síntomas físicos médicamente inexplicables, la consideración de los diagnósticos médicos asociados no resulta sencilla, sin duda adquiere relevancia por las repercusiones en distintos ámbitos de la incapacidad la validez con que goce esta valoración y aunque no existen pautas descritas en estos casos, conviene recordar que no es posible separar la enfermedad del enfermo y que esta sintomatología puede resultar incapacitante. Por supuesto sin obviar que es posible la simulación y el trastorno facticio y que el médico evaluador debe estar en capacidad de identificar estos casos y de establecer o descartar el nexo causal entre una contingencia laboral y el estado secuelar de un paciente.

Ante esto surge la pregunta una vez considerados los aspectos que desde la óptica pericial deben ser enfocados, sobre qué competencias o habilidades clínicas del médico evaluador pueden ser mejoradas en la búsqueda de la validez de los resultados para beneficio del paciente y de la sociedad, concluyendo que resulta trascendental lo siguiente:

a) Formación médica: Debe poseer conocimientos clínicos y biológicos que le permitan realizar una adecuada historia médica y examen clínico del paciente lesionado, con el fin de formular un diagnóstico correcto, relacionándolo con las implicaciones dispuestas en la normativa legal.

b) Conocimientos legales: Conocer el ordenamiento jurídico vigente en torno a las incapacidades laborales.

c) Experiencia: Debe tener práctica profesional en los métodos de valoración de la capacidad funcional laboral y en la utilización de baremos y guías de evaluación en la valoración del daño corporal. Así como en el establecimiento del nexo causal entre los hallazgos y el mecanismo de trauma descrito.

c) Manejo de la Información: Debe estar en capacidad de buscar y recoger información básica en las bases de datos bibliográficas, con el fin de resolver los problemas médico legales que se le planten basándose en la evidencia científica disponible.

d) Capacidad de aprendizaje continuo: Debe estar consciente de sus propias limitaciones teóricas y prácticas y la importancia de mantener y actualizar su competencia profesional, dado el rápido avance de los conocimientos médicos.

e) Razonamiento ético: Debe comprender la trascendencia y obligación legal de su trabajo, no arrojarse la potestad de concluir su dictamen sin tener toda la prueba requerida para hacerlo, incluida la interconsulta psiquiátrica.

Por supuesto, sin obviar que debe ser un buen comunicador, para saber rendir su informe ya sea oral o escrito y fundamentar adecuadamente las conclusiones a las que arriba en su dictamen, que permita entender objetivamente cómo se llegó a ellas.

\section{RECOMENDACIONES}

Como reto futuro, está la investigación de la incidencia con que los diagnósticos asociados a los síntomas físicos sin explicación médica, se presentan en la medicina evaluadora en denuncias por riesgos o enfermedades laborales en Costa Rica, que permita la ulterior comparación con otros estudios y la determinación de los mecanismos de trauma y de en cuántos de ellos, luego del análisis pericial adecuado el médico 
evaluador efectivamente concluye presentan incapacidad para desempeñar su trabajo.

Asimismo resultaría interesante conocer cómo se ha establecido el nexo causal con la contingencia laboral denunciada de ser positivo el resultado, pues de no ser posible establecerlo, aún en condición de incapacidad lógicamente la persona quedaría excluida de atención por parte de la entidad aseguradora, aunque sí seria tributaria de atención por la seguridad social. En todo caso, el médico de la seguridad social también debe considerar la posibilidad de los síntomas físicos médicamente inexplicables para establecer un diagnóstico y tratamiento adecuados.

\section{BIBLIOGRAFÍA}

1. Navarro-Penalver A. Valoración médica de la incapacidad laboral por los facultativos de las Mutuas de Accidentes de Trabajo y Enfermedades Profesionales de la Seguridad Social (MATEPSS). Med Segur Trab (Internet) 2014; 60: 17-24.

2. Bórquez P. Elaboración del informe médico de lesiones. Rev méd Chile 2012; 40: 386-389.

3. Regal-Ramos R. El médico inspector de la Seguridad Social. Med Segur Trab (Internet) 2014; 60:12-16

4. Domínguez-Muñoz A López-Pérez R Gordillo-León F Pérez-Nieto MA Gómez-Álvarez A De la Fuente Madero JL. Bases científicas y bioéticas del análisis de validez en medicina evaluadora. Med Segur Trab (Internet) 2014; 60: 527-535.

5. Moscatello R. Nexo causal: A depressão que gera incapacidade de trabalhar. Revista Consultor Jurídico 2012. Recuperado el 23 de mayo de 2015 en http://www.conjur.com.br/2012-set-05

6. Ezama-Coto E Alonso Y Fontanil-Gómez Y. Pacientes, sintomas, trastornos, organicidad y psicopatología. IJPSY 2010; 10: 293-314

7. Aamland A Malterud K Werner EL. Phenomena associated with sick leave among primary care patients with Medically Unexplained Physical Symptoms: A systematic review. Scand J Prim Health Care 2012; 30: 147-155.

8. Eriksen TE Kirkengen AL Vetlesen AJ. The medically unexplained revisited. Med Health Care Philos 2013; 16:587-600.

9. Huang H McCarron RM. Medically unexplained physical symptoms: Evidence-based interventions. Current Psychiatry 2011; 10: 17 32.

10. Röhricht F Elanjithara T. Management of medically unexplained symptoms: outcomes of a specialist liaison clinic. Psychiatric Bulletin 2014; 38: 102-107.

11. Shraim M Mallen CD Dunn KM. GP consultations for medically unexplained physical symptoms in parents and their children: a systematic review. Br J Gen Pract 2013; e318-e 325.

12. Guzmán-Guzmán RE. Trastorno por Somatización: Su abordaje en Atencion Primaria. Rev Clin Med Fam 2011; 4: 234-243

13. Tófoli LF Andrade LH Fortes S. Somatização na América Latina: uma revisão sobre a classificação de transtornos somatoformes, síndromes funcionais e sintomas sem explicação médica. Rev Bras Psiquiatr 2011; 33:S59-S69.

14. Baeza-Velazco C. Síntoma Psicosomático vs. Sintoma Orgánico: La mala fama de la psicosomática. Cuad Neuropsicol 2010; 4:8-12.

15. Jutel A. Medically unexplained symptoms and the disease label. Social Theory \& Health 2010; 8: 229-245

16. Aamland A Malterud K Werner EL. Patients with persistent medically unexplained physical symptoms: a descriptive study from Norwegian general practice. BMC Family Practice 2014;15:107.

17. Tschudi-Madsen H Kjeldsberg M Natvig B Ihlebaek C Straand J Bruusgaard D. Medically unexplained conditions considered by patients in general practice. Fam Pract 2014; 31: 156-63

18. Stone L. Blame, shame and hopelessness: medically unexplained symptoms and the 'heartsink' experience. Aust Fam Physician 2014; 43: 191-195.

19. Rodríguez-Carvajal R De Rivas-Hermosilla S. Los procesos de estrés laboral y desgaste profesional (burnout): diferenciación, actualización y líneas de intervención. Med Segur Trab (Internet) 2011; 57:72-88.

20. McGorm K Burton C Weller D Murray G Sharpe M. Patients repeatedly referred to secondary care with symptoms unexplained by organic disease: prevalence, characteristics and referral pattern. Family Practice 2010; 27:479_ 486

21. Esteve-Vives J Rivera J Vallejo MA Grupo ICAF. Evaluación de la capacidad funcional en fibromialgia. Analisis comparativo de la validez de constructo de tres escalas. Reumatol Clin 2010;6:141-144. 
22. Medeiros CM Zerbini T Cintra RB Gianvevecchio VAP Muñoz DR. Avaliação da capacidade laborativa em periciandos portadores de fibromialgia. Saúde, Ética \& Justiça. 2012;17:50-6.

23. Castellanos-Ramírez JL. La incapacidad como acto médico. Univ Méd Bogotá 2013; 54 : 26-38.

24. Vicente-Pardo JM. Impacto del dolor en la incapacidad laboral. Metodología de valoración. Grados funcionales de limitación. Med Segur Trab (Internet) 2014; 60: 133-142

25. Martínez-González C González-Barcala FJ Belda-Ramírez J et al. Recomendaciones para la evaluación médica de la capacidad laboral en el enfermo respiratorio crónico. Arch Bronconeumol. 2013;49:480-490

26. Veronesi R. Capacidade funcional para o trabalho: Importante instrumento de decisão para a justiça do trabalho. Interfaces Científicas $2014 ; 2: 23-31$

\section{CONFLICTO DE INTERÉS}

Los autores declaran que no existió ningún conflicto de interés en el presente reporte.

\section{FUENTES DE APOYO}

No hay fuentes de apoyo que declarar. 